Revue internationale de l'économie sociale

Recma

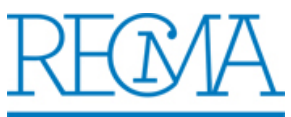

\title{
Le dynamisme associatif en milieu rural, exemple du plateau de Millevaches
}

\section{Christian Vaillant}

Numéro 323, janvier 2012

URI : https://id.erudit.org/iderudit/1018339ar

DOI : https://doi.org/10.7202/1018339ar

Aller au sommaire du numéro

Éditeur(s)

Association Recma

ISSN

1626-1682 (imprimé)

2261-2599 (numérique)

Découvrir la revue

\section{Citer cet article}

Vaillant, C. (2012). Le dynamisme associatif en milieu rural, exemple du plateau de Millevaches. Revue internationale de l'économie sociale, (323), 16-23. https://doi.org/10.7202/1018339ar d'utilisation que vous pouvez consulter en ligne. 


\title{
LE DYNAMISME ASSOCIATIF EN MILIEU RURAL, EXEMPLE DU PLATEAU DE MILLEVACHES
}

\author{
par Christian Vaillant*
}

* Scop La Navette. Mél.: christian vaillant@la-navette.net a connaissance du monde associatif en milieu rural n'a guère mobilisé les chercheurs ces dernières décennies. Quelques travaux du Comité d'étude et de liaison des associations à vocation agricole et rurale (Celavar) et de chercheurs universitaires émaillent les années 90 et 2000, mais aucun ne comporte d'aspects quantitatifs, notamment sur la création d'associations ${ }^{(1)}$. Les seules données récentes disponibles ont été produites par certaines chambres régionales de l'économie sociale (Cres), mais elles portent uniquement sur les associations employeurs, ignorant ainsi plus de $80 \%$ des associations.

\section{Une connaissance subjective}

(1) Agulhon M., Bodiguel M., 1981, Les associations au village, Actes Sud; Celavar, Du rural aux territoires: la contribution des associations, actes du colloque organisé à Toulouse en 2001; Datar et Celavar, 1998, Contribution des associations au développement des services de proximité en milieu rural, Documentation française; Druguet S., 2003, Contributions des associations au développement rural, apports spécifiques et intégration dans la dynamique locale : I'exemple de la Lozère, Centre international de hautes études agronomiques méditerranéennes; Epagneul M.-F., Mathieu N., 2000, "Explorer le rôle des associations dans l'insertion et la création d'emplois en milieu rural ", Economie rurale, $n^{\circ} 259$; Regourd E., 2004, Le phénomène associatif dans la recomposition territoriale: vers de nouvelle ruralité ? université Paul-Valéry Montpellier III; Regourd E., 2007, "Les associations culturelles, porteuses de projet pour de nouvelles ruralités? ", Norois, n 204.
On est donc réduit à une connaissance subjective de la créativité associative en milieu rural et à des hypothèses rationnelles, mais non vérifiées, sur ses déterminants. Il y aurait un plus grand nombre de créations d'association pour deux types de raisons:

- les associations y sont de petite taille et s'organisent au niveau communal pour faciliter leur accès aux habitants (par exemple, une seule association de parents d'élèves, que l'école accueille quinze ou cent cinquante enfants). Dans les villes, l'accès aux associations est plus facile, grâce au réseau de transport et à la proximité géographique, et les associations sont moins nombreuses rapportées au nombre d'habitants;

- un besoin de pallier l'absence ou la faiblesse de l'offre marchande ou publique dans des domaines comme le sport, la culture, les loisirs, etc., ce point étant souvent évoqué en même temps que le rôle des " néoruraux ", plus demandeurs de ce type de services que les " antéruraux».

\section{Une démarche empirique}

Ce travail est d'abord le résultat d'une démarche empirique pour constituer un corps de données à analyser.

Compter le nombre d'associations créées dans une zone rurale (ici le plateau de Millevaches) - dans quelle commune, dans quel secteur 
d'activité, à quelle date - et comparer les données obtenues à celles de l'échelon national constituent le préalable à toute hypothèse. Le plateau de Millevaches se caractérisant fondamentalement par des communes de moins de 1000 habitants et un déclin démographique constant et séculaire, il convenait de mesurer les corrélations éventuelles entre la création associative et ces caractéristiques essentielles. Enfin, il était important de vérifier si à l'homogénéité de cet espace rural correspondait une homogénéité de la création d'associations et quelle était l'éventuelle corrélation avec la présence des néoruraux.

\section{Un relevé systématique des créations d'association sur le plateau de Millevaches}

Cette étude a pour base le relevé systématique dans le Journal officiel des créations d'association dans les douze cantons du plateau de Millevaches de 1986 à 2010 inclus. Ces cantons représentent 114 communes, 47000 habitants et une superficie de $3300 \mathrm{~km}^{2}$. Avec une densité de 14 habitants au kilomètre carré, c'est une des zones les plus rurales de France. Une seule commune (Ussel), en bordure de la zone, atteint les 10000 habitants; les 113 autres comptent moins de 3000 habitants, et 107 moins de 1000.

\section{Un monde rural plus créateur d'associations en apparence seulement}

(2) CNVA, 2003, Bilan de la vie associative en France, 2000-2002, Documentation française; Bazin C., Malet J., 2011, Repères sur les associations en 2010, Recherches et Solidarités; Tchernonog V., 2007, Le paysage associatif français, Juris-Service.
Sur les 114 communes traitées, 1493 associations ont été créées de 1986 à 2010, soit une moyenne de 60 associations par an, soit une association par an pour 788 habitants. La moyenne nationale (2) étant d'une association par an pour 950 habitants, la création d'associations y apparait donc supérieure. Ce phénomène est accentué si l'on excepte Ussel, puisque les 113 autres communes ont alors un taux de création d'une association pour 750 habitants. Ce fort taux est constant sur toute la période.

\section{Des secteurs d'activité très semblables à l'échelon national, avec quelques spécificités}

La répartition des associations par secteurs d'activité dans le plateau de Millevaches n'est pas très différente de la répartition nationale (tableau 1 , en page suivante).

La surreprésentation des associations de loisirs confirmerait l'idée selon laquelle la création d'associations compense l'absence ou la grande faiblesse de l'offre marchande ou publique dans ce domaine. Les difficultés spécifiques à ces territoires en déclin économique expliqueraient aussi l'importance des créations d'associations autour de l'emploi et du développement local et économique.

Enfin, l'apparente faiblesse du secteur sanitaire et social n'est due qu'à l'absence dans le présent recensement des sections et établissements d'associations nationales. Si l'on ajoute les chiffres du Panorama de l'économie sociale en Limousin 2010, publié par l'observatoire de 


\section{Tableau 1}

Répartition par secteurs d'activité

\begin{tabular}{|l|r|r|}
\hline Secteurs & Millevaches & France \\
\hline Sport & $24 \%$ & $24 \%$ \\
\hline Loisirs & $23 \%$ & $18 \%$ \\
\hline Culture & $17 \%$ & $19 \%$ \\
\hline Défense des droits et des intérêts & $13 \%$ & $15 \%$ \\
\hline Professionnelle-Entreprise & $12 \%$ & $4 \%$ \\
\hline Sanitaire et social & $5 \%$ & $11 \%$ \\
\hline Autres & $6 \%$ & $9 \%$ \\
\hline
\end{tabular}

(3) Ce même Panorama recense environ 150 établissements employeurs associatifs, regroupant plus de 1500 salariés, sur le plateau de Millevaches. Le pourcentage d'associations employeurs serait ainsi plus faible sur le plateau de Millevaches que sur I'ensemble de la France (respectivement $10 \%$ et $14 \%$ ), mais le pourcentage de l'emploi associatif apparaîtrait, lui, nettement supérieur à la moyenne nationale ( $14 \%$ contre $8 \%$ ). Ces chiffres, extrapolés, d'une part, des données de l'ensemble de l'économie sociale et, d'autre part, des " pays " ne recouvrant pas exactement les limites cantonales retenues dans notre étude, sont toutefois à prendre avec une grande prudence et ne peuvent représenter que des ordres de grandeur.

(4) Bazin C., Le Vaillant I., Falinower I. et Malet J., 2011, Chiffres clés de la vie associative dans la Creuse, Recherches et Solidarités. l'économie sociale et solidaire du Limousin, concernant le sanitaire et social dans la zone, le pourcentage du secteur atteint alors $10 \%$, très proche du niveau national. Ces établissements (maisons de retraite, foyers occupationnels, instituts médico-éducatifs) sont les principales associations employeurs de la zone et jouent un rôle majeur dans l'emploi. Ils sont dus à l'initiative d'associations nationales, évidemment bien accueillie par les collectivités locales, mais ne témoignent pas de la création associative des habitants du territoire ${ }^{(3)}$. Enfin, une analyse plus détaillée fait apparaître l'importance des associations de chasse et de pêche $(5 \%)$, de défense de l'environnement $(4 \%)$ et des groupements d'employeurs (3\%).

\section{Un monde rural pas si créatif?}

$\mathrm{Si}$, pris globalement, le nombre de créations d'association sur le plateau de Millevaches est plus élevé que la moyenne française, les écarts d'une commune à l'autre peuvent être considérables: de un à quatre ou cinq entre les moins créatives et les plus créatives. Cette diversité se retrouve dans chaque tranche de communes (100 à 150 habitants, 151 à 250, 251 à 400, etc.). On s'aperçoit alors que ce nombre élevé de créations est en réalité porté par un quart des communes, qui créent deux fois plus d'associations que la moyenne nationale. $\mathrm{Si}$ on les retire du calcul, les communes restantes ont un taux moyen identique à celle-ci (presque la moitié font même moins bien). Par ailleurs, la Creuse est l'un des départements les plus ruraux de France, avec 22 habitants au kilomètre carré (14 sur le plateau de Millevaches, mais 115 en France métropolitaine). Selon Recherches et Solidarités ${ }^{(4)}$, le taux de création d'associations, sur les années 2001 à 2010, y est d'une association par an pour 980 habitants, alors qu'il est d'une pour 950 habitants en France. Il se pourrait donc bien 
que, sauf cas très particuliers, le monde rural ne se différencie guère de la moyenne française en termes de créativité associative. Si cette hypothèse se vérifiait, il faudrait peut-être conclure que la créativité associative du monde rural (sauf exception) est moindre que celle du monde urbain, puisque l'intensité de la création est identique alors même que le monde rural bénéficie d'un "avantage objectif ". Cette conclusion ne pourrait évidemment être établie que sous bénéfice d'inventaire. Rappelons en effet que cette hypothèse rationnelle n'est pas étayée par des études. Un simple exemple pris sur le plateau de Millevaches peut éventuellement la relativiser: à Faux-la-Montagne (374 habitants), il y a bien un seul comité des fêtes, comme il n'y en a qu'un dans une ville de 3740 ou 37400 habitants - de même qu'une amicale des pompiers, une association de parents d'élèves (28 élèves...), une association pour les jeunes, etc. -, mais il y a aussi une crèche parentale qui s'étend sur plusieurs communes, une maison des jeux dont l'activité s'étend à tout le plateau, de même que l'association de formation, le Pact-Arim, la maison des associations, la télévision associative locale... Une étude approfondie serait donc indispensable.

\section{Une absence de corrélation avec les caractéristiques démographiques}

\section{Une créativité non corrélée à la taille de la commune...}

La création d'associations paraît difficile dans les communes de moins de 100 habitants (22 communes sur 114) et il semble qu'il y ait là un seuil. La première hypothèse rationnelle devrait d'ailleurs être complétée par ce constat, mais en défaveur de la création d'associations en milieu rural. Au-delà de ce seuil, le niveau de création n'est pas corrélé avec le niveau de population, le nombre de créations par habitant étant très proche quelle que soit la catégorie de communes (de 100 à 150 habitants, de 151 à 250, etc., jusqu'à 1000 à 3000 habitants).

\section{... ni à l'évolution démographique antérieure...}

Le plateau de Millevaches (hors Ussel) perd $22 \%$ de sa population entre le recensement de 1962 et celui de 1982, soit avant le commencement de la période étudiée. On peut émettre deux hypothèses: l'effondrement démographique entraîne soit un découragement général et une baisse de la création d'associations, soit, en réaction, la recherche d'un arsenal de réponses dans lequel figure la création d'associations. Or, il n'existe aucune corrélation entre l'importance du choc démographique et le nombre d'associations créées, ce dernier pouvant être très faible ou très important quel que soit le niveau de cet effondrement. On a ainsi regroupé un lot de communes les plus créatrices d'associations et un lot de communes les moins créatrices. Il apparait alors que la baisse démographique est à peu près la même (respectivement $-20,7 \%$ et $-22,4 \%$ ) et qu'elle est très proche de la moyenne générale $(-21,7 \%)$. 


\section{... ni à l'évolution démographique contemporaine}

Lanalyse de l'évolution démographique 1982-2006 aboutit à un résultat qui peut paraitre paradoxal. Le groupe de communes les plus créatrices d'associations a une perte de population supérieure à la moyenne $(-24 \%)$, tandis que le groupe de communes le moins dynamique subit presque la même évolution que la moyenne $(-15 \%)$. Ce déficit démographique plus grand ne s'explique pas seulement par un solde naturel plus élevé (lui-même s'expliquant uniquement par un nombre de décès plus élevé), mais par un solde migratoire moins positif ( $7 \%$ contre $9 \%$ en moyenne).

Il ne peut y avoir là qưun artefact, car si le dynamisme associatif peut n'avoir qu'un effet faible, voire nul, sur le plan démographique, on ne voit pas pourquoi il aurait un effet négatif. Il serait indispensable de connaitre les composantes du solde migratoire, immigration et émigration, et leurscauses - arrivée de retraités ou d'actifs, par exemple, et de quel type d'actifs -, d'autant que la situation est contrastée: dans ce groupe de communes dynamiques sur le plan associatif, si certaines connaissent un déclin démographique important, d'autres résistent mieux que la moyenne du plateau. En tout état de cause, il n'est pas impossible que, de même que la sur-création d'associations est apparue comme n'étant pas le résultat de la situation et de l'évolution démographique, elle soit également sans effet sur cette même évolution démographique... sauf à penser que, sans lui, cette évolution aurait été encore plus mauvaise.

\section{L'influence des néo-résidents, mais laquelle et lesquels?}

(5) Questions sur l'ancienneté de la «nouvelle " résidence, sur la nature de la résidence d'origine et sur le parcours de vie... auxquelles les données de l'Insee ne répondent pas. En outre, ne sont aisément accessibles que les données complètes des recensements de 1999 et 2006.

(6) Les néo-résidents de moins de cinq ans au recensement de 2006 représentent $11 \%$ de la population. Pour le recensement de 1999 , le pourcentage est de $15 \%$, sachant qu'il s'agit alors de néorésidents de moins de neuf ans. Mais nous ne disposons d'aucune information sur ce que sont devenus depuis ce recensement ces néo-résidents de 1990 à 1999. Nous n'avons retenu que ces deux pourcentages, sans extrapoler le nombre de néo-résidents des deux années manquantes entre les recensements de 1999 et 2006.

\section{Une présence corrélée au nombre de créations d'association}

La définition de "néorural " pose des problèmes méthodologiques et techniques difficiles à surmonter ${ }^{(5)}$ et nous nous en tiendrons au terme de « néorésidents ». Le plateau de Millevaches étant à cheval sur les trois départements du Limousin, nous n'avons retenu comme néo-résidents que les arrivants «hors région Limousin ». En 2006, la proportion des néo-résidents arrivés depuis 1990 est comprise entre 11 et $26 \%{ }^{(6)}$, et elle est probablement beaucoup plus proche du second pourcentage. Mais cette importante proportion de néo-résidents se répartit de manière extrêmement variable selon les communes: de 5 à $50 \%$, compte non tenu des éventuels départs de néo-résidents. La présence des néo-résidents apparaît nettement corrélée positivement à la création d'associations. Si l'on sépare les communes en cinq lots en fonction de l'importance de la présence des néo-résidents, le premier lot (de 30 à $50 \%$ de néo-résidents) a un taux d'une association créée par an pour 620 habitants, le deuxième lot (de 25 à $30 \%$ de néo-résidents) d'une association créee par an pour 690 habitants, soit des taux nettement meilleurs que le taux moyen pour les cinq lots: une association créée par an pour 760 habitants.

La seconde hypothèse rationnelle mentionnée en introduction se vérifie donc. Malheureusement, nous ne disposons d'aucune donnée 
complémentaire sur les néo-résidents - en termes d'âge, de sexe, de catégorie socioprofessionnelle, de niveau de diplôme et de revenus qui permettrait d'affiner l'analyse.

\section{L'éclairage de la cartographie}

La cartographie du dynamisme associatif permet la mise en évidence de zones très différenciées. Cinq degrés d'intensité de création d'associations ont été définis pour classer les 114 communes en cinq lots de 23. Il apparaît alors nettement sur la carte une zone de forte création d'associations à l'ouest du plateau de Millevaches et une zone de faible création à l'est. Plus particulièrement, on relève au nord-ouest un bloc particulièrement créatif de 13 communes (de Royère-de-Vassivière à Saint-Merd-les-Oussines), et la créativité de ces communes est ancienne.

\section{Quels secteurs selon le dynamisme associatif?}

Selon qu'elles appartiennent aux communes les plus créatives ou aux communes les moins créatives, les associations présentent des répartitions par secteurs d'activité nettement distinctes (tableau 2).

Quelques grandes différences apparaissent en effet:

- une très forte proportion des associations de loisirs dans les communes les moins créatrices (lorsqu'il n'y a que deux ou trois associations dans la commune, il y a souvent a minima un comité des fêtes);

- une très faible proportion d'associations culturelles dans ces mêmes communes;

- une très forte proportion des associations autour du développement local et économique dans les communes les plus dynamiques.

\section{Tableau 2}

Dynamisme associatif par secteurs d'activité

\begin{tabular}{|l|r|r|}
\hline Secteurs & $\begin{array}{r}\text { Communes les plus } \\
\text { créatives }\end{array}$ & $\begin{array}{r}\text { Communes les moins } \\
\text { créatives }\end{array}$ \\
\hline Sport & $19 \%$ & $21 \%$ \\
\hline Loisirs & $26 \%$ & $30 \%$ \\
\hline Culture & $16 \%$ & $10 \%$ \\
\hline Défense des droits et des intérêts & $15 \%$ & $12 \%$ \\
\hline Economique & $16 \%$ & $12 \%$ \\
\hline Sanitaire et social & $3 \%$ & $6 \%$ \\
\hline Autres & $6 \%$ & $8 \%$ \\
\hline
\end{tabular}




\section{Le rôle des néo-résidents}

Cet éclairage cartographique permet de reprendre la question des néorésidents. En effet, la zone de 13 communes mise en évidence ci-dessus a, en 2006, une proportion de néo-résidents arrivés depuis 1990 de $31 \%$, ce qui est supérieur à la moyenne du plateau $(26 \%)$. Elle crée deux fois plus d'associations que la moyenne du plateau et deux fois et demie plus que la moyenne nationale. Il se trouve que cette zone a constitué le terrain complet ou partiel de trois études de chercheurs depuis trente ans (1980, 1992 et 2011). Ces trois études décrivent trois générations de néo-résidents présentant les mêmes caractéristiques sociales, professionnelles, culturelles, académiques, ayant les mêmes aspirations (autonomie, agriculture biologique, environnement, coopération...), les mêmes revendications et les mêmes pratiques. Ils sont particulièrement porteurs de projets, d'ailleurs pas seulement associatifs, puisqu'ils explorent toutes les possibilités qu'offre l'économie sociale (coopérative, société à participation ouvrière [Sapo], société coopérative d'intérêt collectif, fonds de dotation,etc.). Ces trois générations sont nommées au fil des études " hippies", "postsoixante-huitards ", " actifs " ou "alters ", caractérisant ainsi les nuances de chaque période.

Ces néo-ruraux, que l'on ne songe plus guère à qualifier de " hippies", n'ont donc pas entre 60 et 70 ans, mais entre 25 et 70 ans. La relation qu'ils ont entretenue avec la population locale, et surtout avec les élus locaux (ce que certains sont d'ailleurs devenus, y compris en tant que maires), en a fait le fer de lance du dynamisme de la commune, particulièrement en matière d'associations. La permanence de leur action a même amené une diversification de la création d'associations: si celles-ci paraissent toujours aussi importantes dans le secteur agricole ou environnemental et dans le développement local, il s'y est ajouté un grand nombre de créations d'ordre "sociétal" (crèche parentale, maison des jeux, bar associatif, covoiturage, bibliothèque...).

\section{Conclusion pour d'autres recherches}

(7) Voir les chiffres contradictoires, et non explicités, d'Insee Première, $n^{\circ} 920$, septembre 2003, et d'Insee Première, $\mathrm{n}^{\circ} 1327$, décembre 2010.
Les quelques résultats de cette recherche soulèvent encore un plus grand nombre de questions, que des travaux futurs pourraient éclairer. Tout d'abord, le "simple " recensement exhaustif des créations d'associations, avec leur secteur d'activité, sur un autre territoire rural permettrait de confirmer, de nuancer ou d'infirmer les conclusions du présent travail.

La première hypothèse rationnelle évoquée en début d'article mériterait d'être approfondie, mais elle pose des problèmes méthodologiques importants: par exemple, la simple question de la taille des associations, même réduite au nombre d'adhérents, est difficile à résoudre, comme le montrent les travaux contradictoires de l'Insee ${ }^{(7)}$; s'y ajoute la difficulté à mesurer la zone d'influence d'une association. Une étude de ce type est 
pourtant indispensable pour savoir si le plus grand nombre de créations d'association en milieu rural n'est qu'apparent, voire masque un dynamisme associatif moindre.

La corrélationétablie avec la présence de néo-résidents mériterait d'être vérifiée et approfondie par des monographies qui permettraient de les caractériser et de savoir si cette sur-création d'associations est due à leur initiative ou à celle d'élus locaux souhaitant les attirer. Le rôle de l'implantation d'une population néo-rurale de type " post-soixante-huitard " renouvelée pourrait être éclairé par une étude sur certaines zones de l'Ardèche, de l'Ariège ou des Alpes-de-Haute-Provence et, inversement, par une comparaison avec une zone rurale ne bénéficiant pas de cette implantation. Ajoutons que l'étude de la structure sociale des différentes communes, l'attitude des habitants de longue date et celle des " néo-ruraux ", les politiques menées par les élus, etc., serait indispensable. Beaucoup de travail en perspective! Mais, en tout état de cause, le dynamisme associatif s'explique avant tout par l'initiative de groupes de personnes plus que par la nature du territoire, celui-ci n'éclairant que les domaines d'activité dans lesquels interviennent les associations créées. 